\title{
Ablation of TRPV4 in HepG2 with Its CRISPR/Cas9 Enhances Its Wound Healing
}

\author{
Eun Jeoung Lee1, Sung Hwa Shin'1, Sunghee Hyun², Sang Sun Kang3* \\ ${ }^{1}$ CdmoGen, Cheongju, Republic of Korea \\ ${ }^{2}$ Department of Pre-Medicine, School of Medicine, Eulji University, Daejeon, Republic of Korea \\ ${ }^{3}$ Department of Biology Education, Chungbuk National University, Cheongju, Republic of Korea \\ Email: *jin95324@cbu.ac.kr
}

How to cite this paper: Lee, E.J., Shin, S.H., Hyun, S. and Kang, S.S. (2020) Ablation of TRPV4 in HepG2 with Its CRISPR/Cas9 Enhances Its Wound Healing. American Journal of Molecular Biology, 10, 74-89. https://doi.org/10.4236/ajmb.2020.101007

Received: December 2, 2019

Accepted: January 7, 2020

Published: January 10, 2020

Copyright (c) 2020 by author(s) and Scientific Research Publishing Inc. This work is licensed under the Creative Commons Attribution International License (CC BY 4.0).

http://creativecommons.org/licenses/by/4.0/

(c) (i) Open Access

\begin{abstract}
TRPV4 activity modulates cell activities including receptor trafficking and transcriptional or translational regulations. We tested its CRISPR/Cas9 scissor efficacy in HepG2 (HEK293) cell noticed that it worked well in both cell lines to eliminate TRPV4 genome sequences. To confirm TRPV4 functions in the cell morphology maintenance and cell growth (beyond $\mathrm{Ca}^{2+}$ channel), we compared its wound healing, cell surface area, survival property and soft agar growth ability after deletion of TRPV4 gene in the cells with its CRISPR/Cas9 system. With these experiments, we confirmed that TRPV4 is required not only to function as $\mathrm{Ca}^{2+}$ channel but also to maintain its proper cell morphology as a corner stone protein on the cell adhesion junction.
\end{abstract}

\section{Keywords}

TRPV4, $\mathrm{Ca}^{2+}$ Ion Channel, E-Cadherin, Tubulin, Soft Agar Growth, CRISPR/Cas9 Scissor System

\section{Introduction}

The TRPV4 channel, a member of the transient receptor potential (TRP) vanilloid subfamily, is expressed in a broad range of tissues where it involves the generation of a $\mathrm{Ca}^{2+}$ signal and/or depolarization of membrane potential [1] [2] [3] [4]. TRPV4 is known as a polymodal receptor that transfers many noxious stimuli into electric signals and is subject to extensive modulation by neurotransmitters, inflammatory cytokines, growth factors, local hormones, and oxidative chemicals, thereby serving as an integration device for processing nociceptive information [4] [5] [6] [7]. The channel contains its distinct cytoplasmic domains to transduce various modulatory inputs into channel activity, which ulti- 
mately adjusts excitability of sensory afferents as a substrate of many cellular signaling pathways [8] [9] [10]. This human TRPV4 gene is found in chromosome12, at q24.1. The gene has 15 exons. C and D: the mRNA consists of 3229 bases, whereas the TRPV4 protein, contains 839 amino [11] [12].

TRPV4 is known to be activated by moderate temperature, endogenous arachidonic acid metabolites, and phorbol esters including the inactive $4 \alpha$-phorbol 12,13-didecanoate (4- $\alpha \mathrm{PDD}$ ), and involved in receptor-operated $\mathrm{Ca}^{2+}$ entry, thus known as polymodal channel. TRPV4 is also stimulated by the mechano- and osmo-transducing messenger 5'-6'-epoxyeicosatrienoic acid (EET). Therefore the role of TRPV4 in osmo- and mechano-transduction contributes to important functions including cellular and systemic volume homeostasis, arterial dilation, nociception, epithelial hydroelectrolyte transport, bladder voiding, and ciliary beat frequency regulation. Ligand binding to each site either directly gates the channel or triggers conformation changes that secondarily modulate thresholds of channel opening by other modalities [13] [14] [15] [16] [17].

To modulate TRPV4 subcellular localization and/or function, several proteins are also identified including microtubule-associated protein 7, calmodulin, with no lysine protein kinases, STIM1, PACSIN3, E-cadherin, N-cadherin, and $\alpha$-catenin, actin, tubulin [17] [18] [19]. In addition, a close functional and physical interaction exists between the inositol triphosphate receptor 3 (IP3R3) and analysis of the gating mechanism of TRPV4 activation for even a single modality is hindered by incomplete understanding of coupling processes among different modes of channel activation [20] [21] [22] [23].

The TRPV4-related pathologies may open a new way to cure the human diseases related with TRPV4 mutations. The mammalian TRPV4 channel has distinct binding sites for small chemical agonists, including endogenous fatty acid or vanilloids, 2-aminoethoxy-diphenyl borate, and purine nucleotides, as well as large macromolecular ligands such as calmodulin or inositide phospholipids. Further, results from animal studies suggest that TRPV4 antagonism has therapeutic potential in oedema, pain, gastrointestinal disorders, and lung diseases such as cough, bronchoconstriction, pulmonary hypertension, and acute lung injury. TRPV4 knockout mice and several synthetic pharmacological compounds that selectively target TRPV4 are now available, which has allowed detailed investigation into the therapeutic potential of this ion channel. TRPV4 mutations are dispersed throughout most domains of the gene. There is no domain-specific phenotype in TRPV4-associated skeletal dysplasias. More than 50 human genetic diseases related with TRPV4 mutations have been reported, including two mutation hot spots (one is the codon for P799 in exon 15, and the other is the codon for R594 in exon 11) [1] [2] [3] [4] [11].

In the course of our work of TRPV4 role in the human cancer cells whether it promotes or not the tumor cell growth, we designed 5 different guide RNA (GR)s for human TRPV4 genome (NM 021625) for the gene scissor of TRPV4 to remove its gene [24]. HepG2 cell was also used to test the gene scissor efficacy 
and noticed its efficacy in HepG2 to eliminate TRPV4 genome sequences. After TRPV4 genome sequences was completely destroyed (or removed $8 \mathrm{bp}$, in theory) with TRPV4 CRISPR/Cas9 system, we compared its wound healing and cell morphology and tumorigenicity, before/after deletion of TRPV4 gene with its CRISPR/Cas9 scissor system to conformed TRPV4 functions in HepG2 cell [25] [26]. Our results suggested that TRPV4 seems to be required to maintain cell attachment with the extracellular matrix/or neighbor cell membrane to block its metastasis and to be necessary for wound healing in HepG2 cell with CRISPR/Cas9 scissor system [27]. Thus, we suggest here that the novel role for TRPV4 has been established as a suppressor protein in HepG2 cell to inhibit metastasis or the epithelial-mesenchymal transition (EMT). Further TRPV4 scissor which seems to be useful as a genetic tool of TRPV4-induced dominant positive human diseases should be also considered more carefully.

\section{Results}

Human TRPV4 removal with its CRISPR/Cas9 scissor (RG7/cas9): The putative structure of TRPV4 (WT, Gene Bank no. BC127052) was shown in Figure 1(a). Transmembrane topology of the mouse TRPV4 (871 aa length). Indicated are the three ankyrin binding repeats (ANK; as gray circles), the six trans-membrane regions (TM1_TM6), the C-terminal cytoplasmic region (718 871 aa) for the $\mathrm{Ca} 2$ / $\mathrm{CaM}$ binding site $(\mathrm{CaM})$, and the $\mathrm{N}$-terminal cytoplasmic region for the putative alpha beta catenin binding site where includes proline

(a)

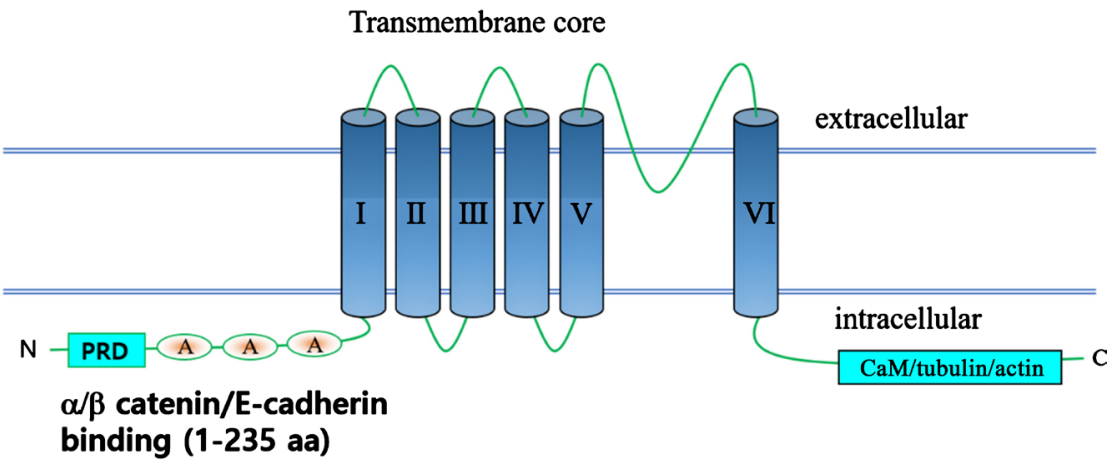

(b)

$$
\text { RG5 : cctggccaatctgtttgaggggg (105-127bp) }
$$

RG6 : ccctttcgecctcaceggctgat (137-159bp) reverse frame

$$
\begin{aligned}
& \text { RG7 : cccaggcgatgggcgaccaaatc (177-199bp) reverse frame } \\
& \text { RG8 : tccagggcg ccttccgcaa gggg (212-234bp) }
\end{aligned}
$$

Figure 1. Human TRPV4 topology and its Scissor guide RNA site. The putative structure of TRPV4 (WT, Gene Bank No. BC127052). (a) Transmembrane topology of the mouse TRPV4 (871 aa length). Indicated are the three ankyrin binding repeats (ANK; as gray circles), the six trans-membrane regions (TM1_TM6), the Ca2_/CaM binding site (CaM). The $\alpha / \beta$ catenin/E-cadherin binding $(1-235$ aa) and the putative cytoplasmic region of TRPV4 (718 - 871 aa) are also indicated. (b) TRPV4 CRISPR RNA guide (RG) sequences and the corresponding DNA sequences site are shown. 
rich domain (PRD) for pacsin binding and ankylin (A) repeat domain. We designed 5 RNA guides for its CRISPR/Cas9 scissor following the guide program [24] [27], and tested its efficacy in HepG2 cell. The corresponding nucleic acid sequence and the frame position are indicated (Figure 1(b)).

The efficacy of TRPV4 Scissor to delete its target gene: To test the ability whether TRPV4 RNA guide gene (RG) directs Cas9-mediated deletion in human HepG2 cells, the mismach sensitive nuclease assay (T7E1 assay) was performed after co transfecting each TRPV4 RG and Cas9 expression vector in the cell (total $1 \mu \mathrm{g}$ dRGEN and Cas9 plasmid in 24 well). The genomic DNA was prepared after $48 \mathrm{~h}$ post-tansfection. Negative control $(\mathrm{N})$ is the PCR product (647 bp PCR product) with PCR primers in Figure 1. Bottom figure represents the reaction without T7E1 for the control of above result. As shown in Figure 2(a), we observed all 4 guide (RG) effectively deleted TRPV4 genome in HepG2 cell. Among them, the most effective RG7 cccaggcgatgggcgaccaaatc 177 - 199 bp; reverse frame was chosen for the next experiment. To determine whether TRPV4 channel protein expression was deleted by RG7 TRPV4 and pGEM Cas9 CMV expression vector, Western blot was performed with the immunoprecipitant with TRPV4 antibody after treatment with RG7 TRPV4 and pGEM Cas9 CMV. As expected, TRPV4 was successfully unexpressed in HepG2 (Figure 2(b) left) which was transfected with RG7 TRPV4 and pGEM Cas9 CMV, compared with the background HepG2 cell (Figure 2(b) right). To confirm further, calcium image experiment was executed. As shown in Figure 2(c), $\mathrm{Ca}^{2+}$ live image in HepG2 cell in the presence of $4 \alpha$-PDD which was used as the TRPV4 specific activator was reduced from $204 \pm 22$ (Arbitrary Unit) to $50 \pm 6$, by TRPV4 scissor treatment. Together these results, the treatment with RG7 TRPV4 and pGEM Cas9 CMV to HepG2 cell successfully ablated its endogenous TRPV4 gene.

HepG2 morphology change by TRPV4 scissor treatment: Previously we demonstrated that the interaction of TRPV4 with actin (or tubulin) is differentially regulated by its 824 serine phosphorylation of TRPV4 [28]. In the line of this observation, we hypothesized that TRPV4 deletion also affects the cell morphology through the alteration of interaction between TRPV4 and cytoskeletal protein such as actin or tubulin. In confocal microscopic observation, we observed that PCC value between tubulin (red) and TRPV4 (green) was $0.68 \pm$ $0.06(\mathrm{~N}=5)$, suggesting the protein-protein interaction between these two proteins is also normally in HepG2 cell (Figure 3(a)). By the way, with TRPV4 RG7 (green)/Cas9 treatment, tubulin (red) subcellular localization was not web network form but it accumulated under the plasma membrane. The cell morphology was also changed as a round shape Moreover, the cell surface area $(2.1 \pm 0.3$ $\left.\mu \mathrm{m}^{2}: \mathrm{N}=5\right)$ was also reduced from the untransfected cell $\left(4.2 \pm 0.2 \mu \mathrm{m}^{2} ; \mathrm{N}=5\right)$. Therefore these results suggested that the deletion of TRPV4 contributes the change of tubulin organization and cell morphology together with E-cadherin expression reduction (Figure 3(d)).

Further, since TRPV4 is also known as a component protein in cell adherent junction to maintain cell morphology [29], next we examine the cell morphological 
(a)

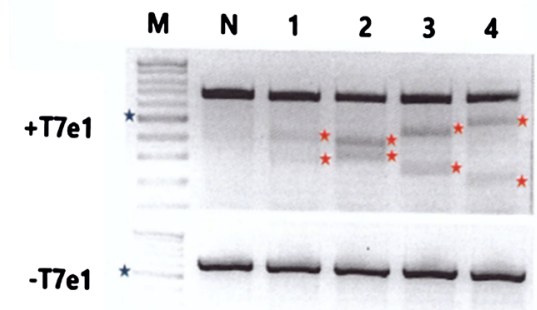

$M$ : PCR add Marker ( $\star 500 \mathrm{bp})$

$\mathrm{N}$ : Negative control

\begin{tabular}{|c|c|c|c|c|}
\hline Gene & Total size & RG & \multicolumn{2}{|c|}{ Fragment } \\
\hline & & 5 & 355 & 292 \\
\hline \multirow{3}{*}{ TRPV4 } & 647 & 6 & 376 & 271 \\
\hline & & 7 & 416 & 231 \\
\hline & & 8 & 462 & 185 \\
\hline
\end{tabular}

(b)

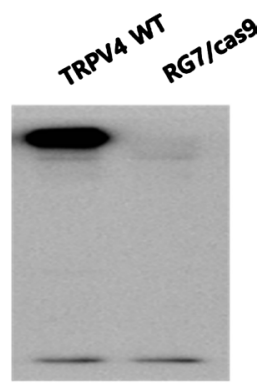

(c)

Optical density
(Arbitrary Unit)
$125+/-22$

300
250
200
150
100

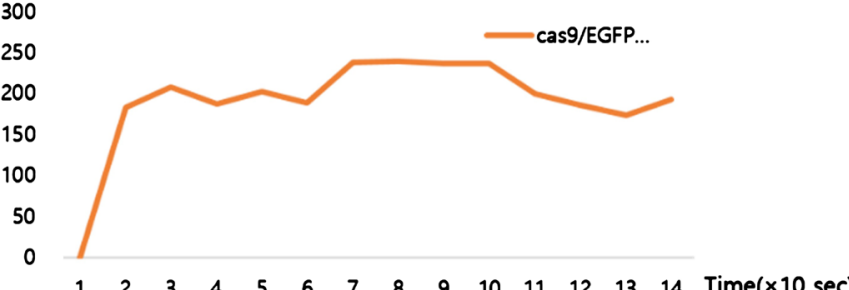

No treatment Cas9 TRPV4 RG7 +4 a PDD

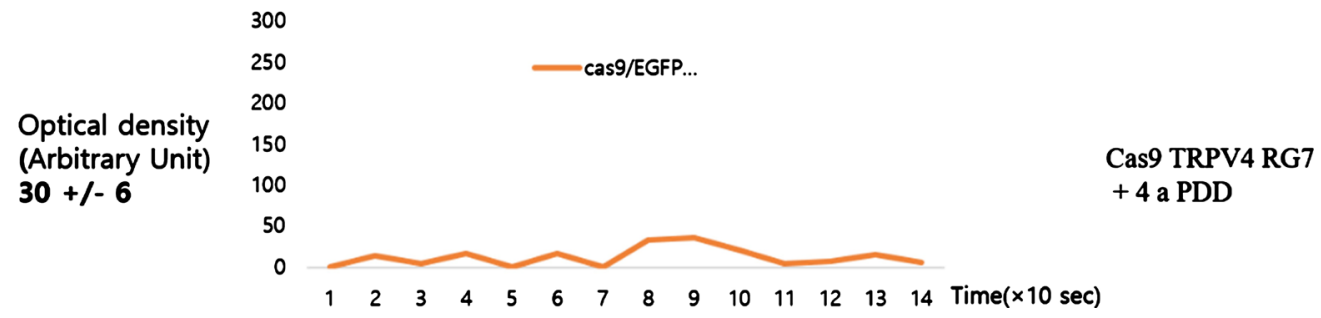

Figure 2. Mismatch sensitive nuclease assay (a) and western blot (b) and $\mathrm{Ca}^{2+}$ live image (c) in HepG2 cell after TRPV4 Scissor treatment. (a) To test the abilities of TRPV4 RNA guide gene (RG) to direct Cas9-mediated deletion in human HepG2 cells, we performed the mismach sensitive nuclease assay (T7E1 assay), after cotransfecting TRPV4 RG and Cas9 expression vector in the cell (total $1 \mu \mathrm{g}$ dRGEN and Cas9 plasmid in 24 well). The genomic DNA was prepared at $48 \mathrm{~h}$ post-tansfection. The star maker represents the expected cut pattern according to each RG with T7E1 assay (left table). N: Negative control is the PCR product (647 bp PCR product) with primers in Figure 1. Bottom figure represents the reaction without T7E1 for the control of above result. (b) HepG2 cells which express their endogenous TRPV4 were transiently transfected with EGFP-TRPV4 WT and Cas9 plasmid. After $48 \mathrm{~h}$ the cells were lysated, and immunoprecipitation was conducted with mouse anti-TRPV4 antibody. Western blot assays were performed with rabbit anti-TRPV4. The endogenous TRPV4 (MW: 130 Kd) of HepG2 cell (left lane) was undetectable in TRPV4 RG7/Cas9 treated cell (right lane). (c) In HepG2 cells, the endogenous TRPV4 was successfully deleted by treatment of TRPV4 RNA guide gene (RG) and Cas 9 expression. $\mathrm{Ca}^{2+}$ live image in HepG2 cell in the presence of $4 \alpha$ PDD was reduced from $204 \pm 22^{*}$ (Arbitrary Unit) to $50 \pm 6^{*}$, through TRPV4 scissor treatment (after one day later). ${ }^{*} p$ value less than 0.05 .

change and E-cadherin subcellular localization after deletion TRPV4 with its gene scissor (Figure 3(c)). Consistent with the above results (Figure 3(b)), the average surface area of the green colored cells which were affected by TRPV4 RG7 (green)/Cas9 was reduced as a half of that of untransfected cells. Furthermore, the surroundings of green colored cell were not attached with E-cadherin (red), while that of untransfected cells were attached with E-cadherin (red). These results suggested that E-cadherin expression was down regulated in green colored cell which was transfected with TRPV4 RG7/Cas9. Together, these results suggested that not only TRPV4 RG7 (green)/Cas9 treatment was successfully operated in HepG2 cell, but also the deletion of TRPV4 resulted in the cell morphology 
(a)

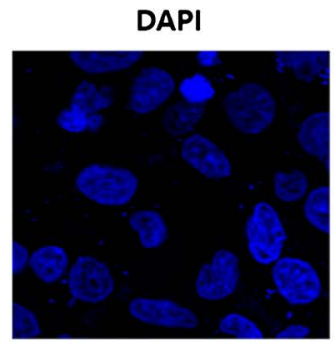

DAPI

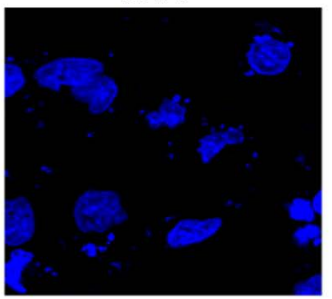

DAPI

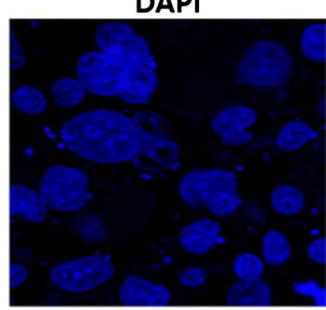

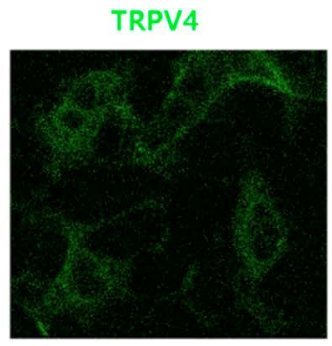

TRPV4 RG7/Cas9

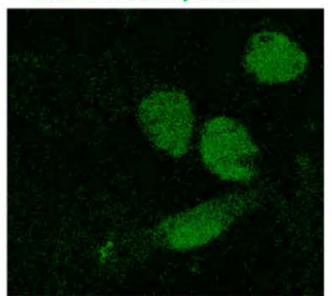

TRPV4 RG7/Cas9

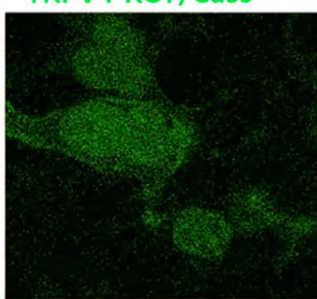

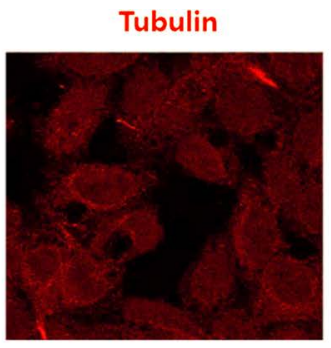

Tubulin

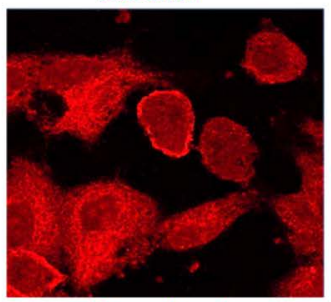

E-cadherin

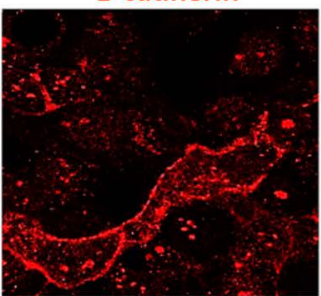

Blue color (Transfected) cell surface area $\left(2.1 \mu \mathrm{m}^{2}: \mathrm{N}=5\right)$ Un-transfected cell $\left(4.2 \mu \mathrm{m}^{2} ; \mathrm{N}=5\right)$

HEK 293 TRPV4 RG7/Cas9

E- cadherin mouse $\mathrm{Ab}$

(d)

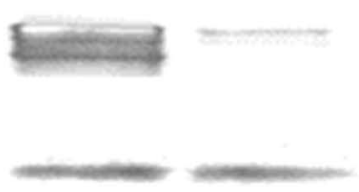

Figure 3. Tubulin and E-cadherin subcellular localization change in HepG2 cell by TRPV4 Scissor treatment. (a) Confocal microscope images $(\times 400)$ show that EGFP-TRPV4 WT (green) and tubulin (red) was merged (yellow). PCC was $0.68 \pm 0.06(\mathrm{~N}=5)$. DAPI staining represents the nuclear region (blue). (b) After TRPV4 RG7 (green)/Cas9 treatment, tubulin (red) subcellular localization and cell surface area were measured with the confocal microscopy. The cell morphology became round shape and the tubulin accumulated the under plasma membrane, instead of the web net connection. (c) The extracellular liner accumulation of E-cadherin was disappeared after TRPV4 RG7/Cas9 treatment. The cell surface area $\left(2.1 \pm 0.3^{*} \mu \mathrm{m}^{2}: \mathrm{N}=5\right)$ was also reduced from the untransfected cell $\left(4.2 \pm 0.5^{*} \mu \mathrm{m}^{2}\right.$; $\mathrm{N}=5$ ). ${ }^{*} p$ value less than 0.05 . (d) After immunoprecipitation with an anti-E cadherin rabbit Ab, western blot was performed with the anti E cadherin mouse Ab, with TRPV4 RG7/Cas9 treatment. With TRPV4 genetic scissor treatment, the endogenous $\mathrm{E}$ cadherin expression (measured by a densitometer) was reduced to 1/30, approximately. The negative control (-) was the immunoprecipitant with an unrelated rabbit $\mathrm{Ab}$.

change to the round shape and the reduction of cell surface area to a half, by mean of the tubulin and E-cadherin organization change (Figure 3(c)). Further, with western blot analysis, we also observed the dramatic reduction of E-cadherin expression (approximately 1/30) by means of TRPV4 RG7 (green)/Cas9 treatment (Figure 3(d)). Therefore, TRPV4 seems to be the major corner stone protein for its plasma membrane attachment with other cytoskeletal proteins including E-cadherin and tubulin thereby providing a link to the actin cytoskele- 
ton in Figure 5 [14] [17] [28] [29] [30].

The enhancement of cell growth, soft ager growth ability and wound healing by TRPV4 scissor treatment: To determine whether the elimination of interaction between TRPV 4 and E-cadherin (actin, $\alpha / \beta$ catenin or tubulin) promotes its cell growth, soft agar growth ability and wound healing [28] [29], we compared each ability of TRPV4 scissor treated HepG2 cells with that of background cell.

After TRPV4 gene scissor (EGFP RG7/Cas9), or EGFP vector was transfected, the rate of apoptosis was measured by FACS (Table 1). Unexpectedly, the apoptotic effect of TRPV4 gene scissor was reduced to a half that of the EGFP vector alone transfected cell (Table 1). Because the removal of TRPV4 genome in HepG2 cell by TRPV4 gene scissor promotes the cell growth, the role of TRPV4 seems to be an anti-growth gene. To access further the role of TRPV4 in the cell growth, we measured the effect of TRPV4 activity on the wound healing with the transfected HepG2 cell with TRPV4 RG7/Cas9 (Figure 4(d)). Surprisingly, we observed that the wound steak width of untreated HepG2 was $6.2 \pm 0.2(\mathrm{~N}=5)$,

Table 1. The comparison of cell survival ratio of HepG2 with its gene scissor treated Cell.

\begin{tabular}{cc}
\hline TRPV4 & Rate of apoptosis (\%)by FACS \\
\hline Non treatment & $14.73 \pm 2.52$ \\
RG7/CAS9 & $7.16 \pm 1.76^{*}$ \\
Vector only & $14.69 \pm 1.75$ \\
& Mean value of 5 repeats \\
\hline
\end{tabular}

${ }^{*} p$ value less than 0.05. TRPV4 gene scissor (EGFP RG7/Cas9), or EGFP vector was transfected and the rate of apoptosis measured by FACS. HepG2, EGFP-TRPV4 RG7/CAS9 were compared to EGFP vector alone constructs. The apoptotic effect of TRPV4 gene scissor was reduced to 2.0 times that of HepG2 cell.

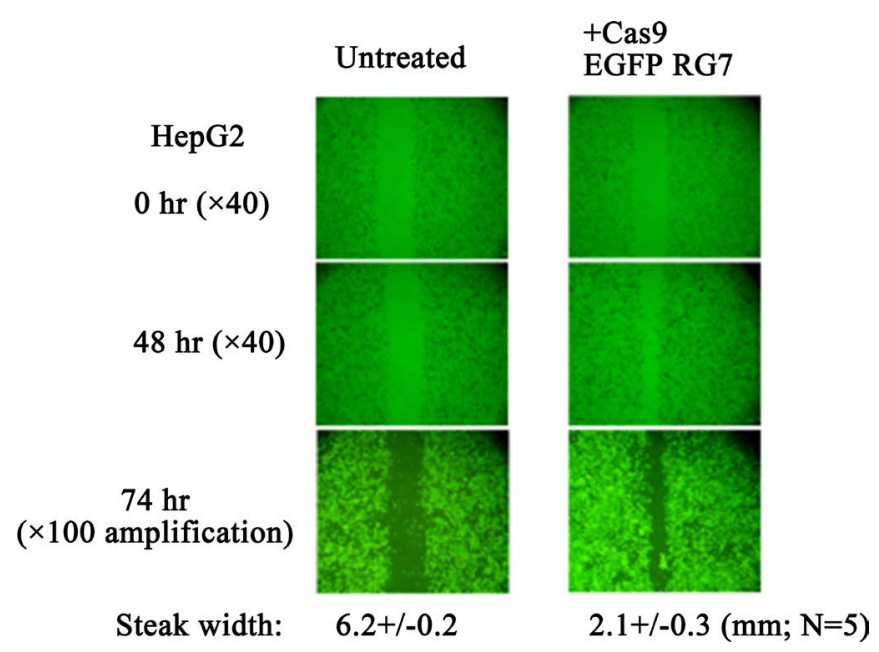

Figure 4. HepG2 wound healing after TRPV4 Scissor treatment. The effect of TRPV4 activity on cell growth was determined by the wound healing analysis with the transiently transfected HepG2 cell with TRPV4 RG7/Cas9. Steak width was reduced according to the incubation time. After $72 \mathrm{hr}$ incubation, the steak width of untreated HepG2 was $6.2 \pm$ $0.2^{*} 2.1 \pm 0.3^{*} \mathrm{~mm}(\mathrm{~N}=5)$, that of transfected HepG2 with TRPV4 RG7/Cas9 was $2.1 \pm$ $0.3^{\star}(\mathrm{mm} ; \mathrm{N}=5)$. Magnitude $\times 40(0$ and $48 \mathrm{hr}) ; \times 100(72 \mathrm{hr}){ }^{*} p$ value less than 0.05 . 
that of transfected HepG2 with TRPV4 RG7/Cas9 was $2.1 \pm 0.3(\mu \mathrm{m} ; \mathrm{N}=5)$ after $72 \mathrm{hr}$ incubation, suggesting that the transfected HepG2 with TRPV4 RG7/Cas9 grew 3 time faster than the untreated cell did (Figure 4).

In order to define TRPV4 role in the tumor, we compared the soft agar growth (colony forming) ability of TRPV4 gene scissor treated cell with its background HepG2 cell (Table 2). TRPV4 gene scissor (EGFP TRPV4 RG7/Cas9), or EGFP vector was transfected and the growth (colony forming) ability on the soft agar after 500 cells plating. After 3 week, we detected that only the cells treated with TRPV4 gene scissor formed the cell colony on the soft agar $(7 \pm 2 ; \mathrm{N}=5)$. Because the deletion of TRPV4 promotes the malignancy of HeG2 cell, the channel seems to play a role as tumor suppressor protein [28] [29].

In summary, with TRPV4 gene scissor, we demonstrated here the presence of TRPV4 roles as a corner stone to maintain the native pseudopod shape and it inhibits cell extravasation and EMT. Therefore, TRPV4 plays a key role as a corner stone to assemble the cytoskeleton such as tubulin or microtubule in the plasma membrane to maintain cell proper shape. The interaction between TRPV4 and the cytoskeletal protein (actin, $\alpha / \beta$ catenin or tubulin) regulates the proper cell shape or EMT through E-cadherin expression reduction (Figure 5).

\section{Discussion}

TRPV4 was originally shown to be activated by hypotonicity, but later studies have demonstrated that activation can also be achieved by phorbol esters, arachidonic acid, and moderate heat. TRPV4 appears to be an important player in pathological sensory perception and bone growth. The potential effect of a TRPV4 functional mutation remains to be elucidated. Furthermore, the role of TRPV4 in the pathogenesis of several diseases should be characterized as to how the channel protein contributes to the specific diseases. Further, TRPV4 is expressed at a higher level in tumor-derived endothelial cells when compared with normal endothelial cells. To cure or alleviate the human disease caused by TRPV4 mutations the information may be useful. Recently, other researcher reported that the antagonism of TRPV4 inhibits both matrix stiffness- and TGF $\beta 1$-induced the epithelial-mesenchymal transition (EMT) in normal human

Table 2. The comparison of soft agar growth (colony forming) ability of TRPV4 gene scissor treated cell with its background HepG2 cell.

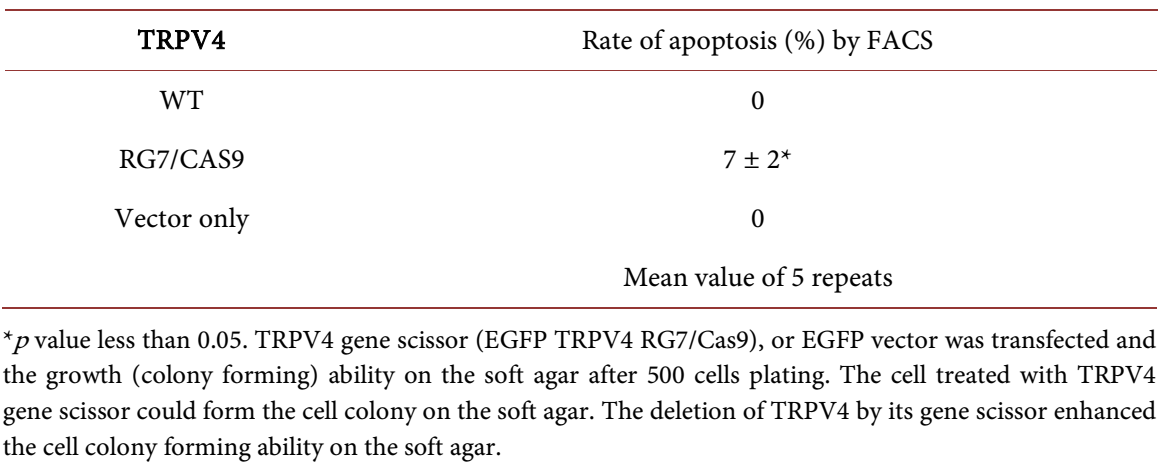




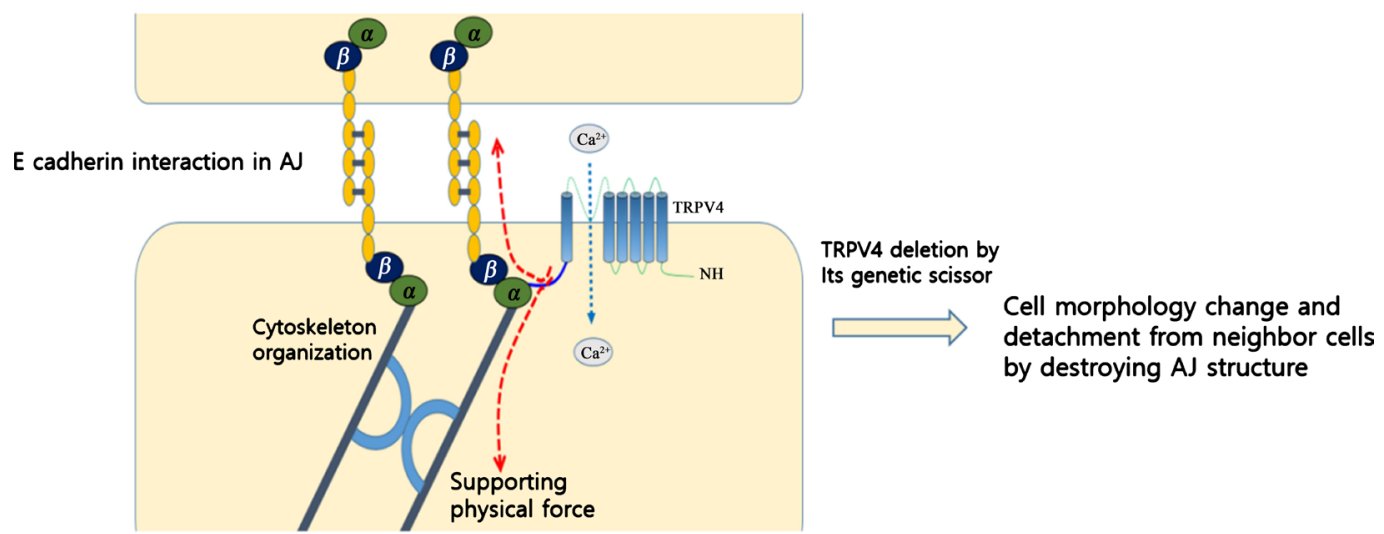

TRPV4 functions:

- $\mathrm{Ca}^{2+}$ channel

- Physical connecting protein between E-cadherin of AJ and intracellular cytoskeleton protein on plasma membrane to maintain cell shape

- Inhibition of cell migration

Figure 5. TRPV4 function on cell adhesion junction as a corner stone: TRPV4 seems to play a role as a corner stone to assemble the cytoskeleton such as tubulin or microtubule in the plasma membrane. The interaction between E-cadherin, tubulin and TRPV4 was regulated by the protein-protein interaction and the posttranslational modification for the proper cell shape or the epithelial-mesenchymal transition. Without TRPV4, cells became round shape and detached from the adhesion junction of the neighbor cell, caused by E-cadherin disorganization. Functions of TRPV4 are summarized as three points in the below.

primary epidermal keratinocytes, as determined by changes in morphology and alterations in expression of EMT markers including E-cadherin, N-cadherin [30]. However, our results support that the expression of TRPV4 reduces both EMT and tumorigenesis (Table 2).

Mutations of TRPV4 result in both a range of skeletal dyplasias and peripheral neuropathies. Mutant TRPV4 can result in skeletal dysplasia and degenerative neuropathy in the same patient. TRPV4 mutations expected to result in "neuropathies" can also cause skeletal dysplasias while TRPV4 mutations of the "skeletopathic" type can also result in neuropathies [1] [2] [3] [4]. Other researcher study did, however, concede that the "gain of function" of the mutant channels could be the result of more effective channel insertion of mutant TRPV4 proteins. However, the data in other study of CMT $2 \mathrm{C}$ also supports the proposition that "gain of function" of mutant TRPV4, rather than "loss of function" of these mutant channels, is pathologically important [4] [11]. Both mutant TRPV4 receptors have normal physiological localization within the cells expressing them. Cells transfected with these mutant TRPV4 receptors have elevated intracellular $\mathrm{Ca}^{2+}$ levels, and cell death can be reversed by ruthenium red. Thus, it still seems to be controversial how TRPV4 mutation contributes cell growth. Therefore, many results suggested that the mechanism of action of the TRPV4 mutants involves a "gain of function" of the ion channel likely to lead to increased intracellular $\mathrm{Ca}^{2+}$ concentration.

Among many TRPV4 mutants, the two hot spot mutants (V620I, P799R) cause the metatropic dyslpasia by the gain function [1] [2] [3] [4]. The result 
seems to be varied, depending on the cell line which is used in experiment and experiment condition. Even though many TRPV4 point mutant humans suffer many genetic diseases, the TRPV4 knockout mouse has no obvious symptom. Thus, we rationalize that the "gain of function" of mutant TRPV4 can be cured by the genetic scissor to remove the point mutant TRPV4 which caused each specific human genetic disease. Therefore, to remove TRPV4 genome, we design the specific TRPV4 genetic scissor with the several guide RNA expression vector (Figure 1(b)). Subsequently, we succeed to remove TRPV4 genome in HepG2 cell HEK293 and HCT116 (colon cancer cell line: data not shown). Consequently, we assume that the "gain of function" of mutant TRPV4 can be cured by the genetic scissor to remove the mutant TRPV4.

Recently, researchers revealed that TRPV4-expressing cancer cells are softer and that TRPV4-conferred cell deformability was associated with actin depolymerization, VASP phosphorylation and inversely correlated with the activation of ERM and Cofilin [29]. However, our observation suggested that the deletion of TRPV4 enhances its wound healing, cell deformability, soft agar growth, and metastasis. Therefore, upregulation of TRPV4 seems to be anti-cancer protein in our system.

Other researchers also reported that the down regulation of TRPV4 is a key determinant of tumor endothelial cell tumor angiogenesis and abnormal vasculature [30] [31] [32]. This study also supports a role for TRPV4 in metastasis by regulating cancer cell stiffness and cytoskeleton at the cell cortex. Our result seems to be support TRPV4 role in the metastasis inhibition by the strength its adhesion junction (Figure 5). However, further studies will be required to clarify the TRPV4 role in metastasis. To test this hypothesis further, we are comparing now the tumor metastasis in TRPV4 knockout mouse with that in WT mouse.

We subsequently measured cell surface area expansion with TRPV4 scissor treatment to access whether TRPV4 knockdown affects cell shape or morphology. As compared TRPV4 scissor, the cell surface area of WT remained unchanged, whereas that of was significantly increased. It has been characterized that TRPV4 links to E-cadherin through catenin. So we expected that if TRPV4 was deleted in the cell by its scissor, the cell adhesion with E-cadherin was also blocked. As expected, the transfected cell with TRPV4 scissor (green color) was not attached with E-cadherin and the cell morphology was round shape. Therefore, TRPV4 seems to contribute the cell shape formation as the corner stone on the plasma membrane. TRPV4 is characterized as the mechanoreceptor in bladder urothelium and skin epithelial cell. To do that, it should connect with cytoskeletal protein such as actin and tubulin. The N-terminal of TRPV4 is responsible to associated with $\mathrm{a} / \mathrm{b}$ catenin proteins [1]. We observed that the web matrix formation of tubulin was destroyed by TRPV4 scissor treatment, and the tubulin in the cell was localized in the edge of cell. Therefore, the results also support that TRPV4 as the corner stone on the plasma membrane contributes as the mechanoreceptor which associates with the cytoskeletal proteins. Once again, the confocal re- 
sults confirmed that TRPV4 scissor can work properly to remove the target genome.

TRPV4 is expressed at a higher level in tumor-derived endothelial cells when compared with normal endothelial cells [25]. TRPV4 is important in the migration of the former cell type but not in relation to the latter. This, in turn, causes elevation of the level of TRPV4 found in the plasma membrane. This increased level of TRPV4 in the plasma membrane is believed to be important in promoting endothelial cell migration which results in angiogenesis and tumor growth. On the other hand, the level of TRPV4 found in healthy or inflamed skin keratinocytes is reduced in premalignant lesions and in non-melanoma skin cancer, although whether this is a component of the mechanism of the disease remains unknown.

TRPV4 seems to be down-regulated during metastasis to detach from the basal attachment with extracellular matrix or neighbor cell (Figures 3-5). Therefore our results support that TRPV4 seems to be one of anti-metastatic $\mathrm{Ca}^{2+}$ channel (Table 1 and Table 2). We assumed that the down regulation of TRPV4 seems to promote the release of $\alpha / \beta$-catenin from the adherent junction, and the free $\beta$-catenin which is translocated to the nuclear stimulates the cell proliferation, like Wnt signaling (Figure 5). In addition, because the use of TRPV4 CRISPR/Cas9 scissor has been shown to remove out TRPV4 genome successfully in HEK293 and HCT116 (colon cancer cell line: data not shown) cell, this scissor can be also useful to genetic treatment of TRPV4-induced dominant positive diseases with its gene scissor. However, we cannot rule out the possibility that TRPV4 crisper/cas9 delete the tumor suppressor gene during its working in the cell and cell became more mutagenesis.

The epithelial-mesenchymal transition (EMT) is defined as a process by which epithelial cells lose their cell polarity and cell-cell adhesion, and gain migratory and invasive properties to become mesenchymal stem cells; these are multipotent stromal cells that can differentiate into a variety of cell types. Thus, EMT is essential for numerous developmental processes including mesoderm formation and neural tube formation. EMT has also been characterized to occur in wound healing, in organ fibrosis and in the initiation of metastasis in cancer progression. Because TRPV4 removal by its genetic scissor enhanced HepG2 cell wound healing and its soft agar growth through the downregulation or disrupt E-cadherin organization (Figure 3 and Figure 4, Table 1), the channel protein seems to be involved in the EMT process. Therefore, because TRPV4 seems to be a target protein to be downregulated for the EMT, TRPV4 may be regarded as a tumor suppresser protein (Figure 5).

Because the deletion of endogenous TRPV4 by its scissor enhances its tumorigenicity in breast and colon cancer, the proper TRPV4 function seems to be required for its antitumor activity. TRPV4 is expressed at a lower level in tumor-derived endothelial cells when compared with normal endothelial cells [25] [26]. TRPV4 which is defined as a $\mathrm{Ca}^{2+}$ channel functions as not only the physi- 
cal connecting protein between E-cadherin in $\mathrm{AJ}$ and intracellular cytoskeleton protein on plasma membrane to maintain cell shape but also the inhibitor on the liver cancer metastasis [23] [29] [30]. However, we assumed that TRPV4 deletion contributes to the cell growth by increasing free cellular-catenin which is otherwise hold by TRPV4 under the plasma membrane. The deletion effect of TRPV4 activity with was also determined by whole-cell $\mathrm{Ca}^{2+}$ image analysis in transiently transfected HepG2 cells. Even though the regulation of cell stiffness and cell cortex dynamics are putative modes of actions through which TRPV4 promotes cancer cell extravasation, the upregulation of TRPV4 may be a viable drug target for management of metastatic liver cancers [29] [30].

In conclusion, we demonstrated here with TRPV4 CRISPR/Cas9 system that the channel which plays role as $\mathrm{Ca}^{2+} / \mathrm{Mg}^{2+}$ ion transporter, also has a role for the corner stone to connect physically between E-cadherin and the intracellular cytoskeleton protein with the plasma membrane to maintain cell shape (Figure 5). In addition, TRPV4 functions as the suppressor of metastasis or EMT to maintain the integrate cell shape, with attaching the neighboring cell in AJ.

\section{Materials and Methods}

Cell Culture: HepG2 was cultured with DMEM supplemented with 10\% newborn calf serum and antibiotics. Cells were transfected with $400 \mathrm{ng}$ wild-type or mutant receptors with or without 50 ng enhanced GFP plasmids using Turbofect reagents (Fermentas).

Construction TRPV4 Gene Scissor: TRPV4 were constructed in a mammalian expression vector, following the instruction of Gene Tool Co (Seoul Korea) and were designed four guide RNA (RG)s to delete TRPV4 in HEPG2 cell, corresponding to GenBank DQ059646.1. RG5: cctggccaatctgtttgaggggg (105 - 127 bp) RG6: ccctttcgccctcaccggctgat (137 - 159 bp) reverse frame RG7: cccaggcgatgggcgaccaaatc (177 - 199 bp) reverse frame GR8: tccagggcg ccttccgcaa gggg (212 - 234 bp) which were indicated in Figure 1(b) were expressed in Tool Gene vector (pRGEN human TRPV4 U6SG) with pGEM Cas9 CMV expression vector (Supplement 1).

Western Blotting: Cells transfected with cDNA-containing plasmids were grown in cell culture plates until $48 \mathrm{~h}$ after transfection. Cells were rinsed three times with PBS. The buffer solution was gently removed and cells were lysed in PBS containing $1 \%$ Triton X-100 and protease inhibitor mixtures (Thermo Scientific). To test the reversibility of covalent dithiaarsanane adducts, we included $10 \mathrm{~mm}$ mercaptoethanol in the lysis buffer. The lysate was resolved on $8 \%$ non-reducing SDS-polyacrylamide gel, which was transferred to an Immobilon membrane (Millipore). The membrane was incubated in blocking buffer (Tris-buffered saline with $0.05 \%$ Tween 20 with $5 \%$ nonfat milk) containing anti-HA antibody (at 1:1000 dilution, Covance, Inc.) at $4^{\circ} \mathrm{C}$ overnight. TRPV4 proteins were visualized using a secondary goat-anti-rabbit HRP-conjugated antibody at 1:100,000 dilution (Thermo Scientific).

Fluorescence Measurements of $\left[\mathrm{Ca}^{2+}\right] \mathrm{I}$ : We measured $\left[\mathrm{Ca}^{2+}\right] \mathrm{i}$ using a fluores- 
cent $\mathrm{Ca}^{2+}$ indicator Fluo4-acetoxymethyl ester (Fluo4-AM), as previously described. In brief, cells growing on coverslips were incubated for $40 \mathrm{~min}$ in DMSO solution containing $1 \mu \mathrm{M}$ Fluo4-AM at $24^{\circ} \mathrm{C}$ in darkness, and then washed and incubated for $15 \mathrm{~min}$ to hydrolyze internalized Fluo4-AM. We measured $\left[\mathrm{Ca}^{2+}\right] \mathrm{i}$ in single cells that emitted fluorescence, using confocal microscopy (LSM710 Zeiss, Germany) at wavelengths of $495 \mathrm{~nm}$ (excitation), and $519 \mathrm{~nm}$ (emission). The absorption (as an arbitrary unit) at $488 \mathrm{~nm}$ with an argon-ion laser was measured as a relative intracellular $\mathrm{Ca}^{2+}$ ion concentration $\left[\mathrm{Ca}^{2+}\right]$ i. All experiments were carried out at $24^{\circ} \mathrm{C}$. After stimulation with mild heat (from $24^{\circ} \mathrm{C}$ to $42^{\circ} \mathrm{C}$ within $45 \mathrm{~s}$ for 2 $\min ),\left[\mathrm{Ca}^{2+}\right]$ i was measured in single cells at a $24^{\circ} \mathrm{C}$ solution.

Confocal Microscopy: HepG2 cells were seeded overnight at $60 \%$ confluence onto culture slides coated with human fibronectin (Becton Dickinson, MA). The cells were washed several times with ice-cold PBS and fixed in 3\% paraformaldehyde for 10 minutes. The fixed cells were permeabilized with $0.1 \%$ Triton X-100 for 10 minutes and blocked for 1 hours in PBS containing 5\% BSA (Sigma, USA) and $0.1 \%$ Tween. Following incubation with a polyclonal antibody against E-cadherin or monoclonal antibody against TRPV4, the cells were washed and stained further with a conjugated donkey anti-rabbit IgG prior to processing the slides for immunofluorescence. After an additional 20 minutes of incubation at $37^{\circ} \mathrm{C}$, the cells were fixed, permeabilized, and decorated with either an anti-E-cadherin or TRPV4 antibody. As a secondary antibody, Alexa Fluor 568-conjugated donkey anti-rabbit or Fluor 488-conjugated goat anti-mouse (Molecular Probes, Inc., Eugene, OR) was used. Confocal microscopy analysis was performed LSM710 (Zeiss, Germany) at the Center for Experimental Research Facilities of Chungbuk National University. Cell surface area was also determined with the confocal microscopy analysis.

Solutions and Drugs: Cells were superfused normally with a solution containing (mM): $88 \mathrm{NaCl}, 5 \mathrm{KCl}, 5.5$ glucose, $1 \mathrm{CaCl}_{2}, 10$ HEPES and 100 mannitol, adjusted to $\mathrm{pH} 7.4$ with $\mathrm{NaOH}\left(300\right.$ mosm $\mathrm{kg} \mathrm{H}_{2} \mathrm{O}$ ). The HTS was adjusted by omitting mannitol. $4 \alpha$-PDD was acquired from Sigma (St Louis, MO, USA). Fluo-4AM was acquired from Molecular Probes, Inc (Eugene, OR, UK). Stock solutions of phorbol esters were initially prepared in dimethyl sulphoxide (DMSO) at a concentration of $1 \mathrm{mM}$, and then stored at $-20^{\circ} \mathrm{C}$. The final DMSO concentration in the experimental bath solution containing phorbol esters never exceeded $0.5 \%$. Insulin and TRPV4 inhibitors GSK 650394 were acquired from Tocris Bioscience (Ellisville, MO, USA), and used them as the manufacturer's recommendation.

Statistical Analysis: Data were presented as mean \pm S.E. A $p$ value less than 0.05 was considered to be statistically significant.

Background: TRPV4 channel requires maintaining cell morphology of HepG2 cell.

Results: Deletion of TRPV4 with CRISPR/Cas9 scissor changes HepG2 cell morphology.

Conclusion: The ablation of TRPV4by CRISPR/Cas9 system enhances HepG2 
soft agar growth and wound healing by alteration of cytoskeletal protein organization.

Significance: TRPV4 contributes to maintain its proper cell morphology and migration as a cornerstone protein

\section{Acknowledgements}

This work was supported by a National Research Foundation of Korea grant (NRF-2013R1A1A4A01005522 and NRF-2016R1D1A3B03934269) to S. S. Kang. Lee is Korean Research fellowship recipients (NRF-2017R1A6A3A11032094).

\section{Conflicts of Interest}

The authors declare no conflicts of interest regarding the publication of this paper.

\section{References}

[1] Grace, M.S., Bonvini, S.J., Belvisi, M.G. and McIntyre, P. (2017) Modulation of the TRPV4 Ion Channel as a Therapeutic Target for Disease. Pharmacology \& Therapeutics, 177, 9-22. https://doi.org/10.1016/j.pharmthera.2017.02.019

[2] Kang, S.S., Shin, S.H., Auh, C.K. and Chun, J. (2012) Human Skeletal Dysplasia Caused by a Constitutive Activated Transient Receptor Potential Vanilloid 4 (TRPV4) Cation Channel Mutation. Experimental \& Molecular Medicine, 44, 702-722. https://doi.org/10.3858/emm.2012.44.12.080

[3] Nilius, B. and Owsianik, G. (2010) Channelopathies Converge on TRPV4. Nature Genetics, 42, 98-100. https://doi.org/10.1038/ng0210-98

[4] Nilius, B. and Voets, T. (2013) The Puzzle of TRPV4 Channelopathies. EMBO Reports, 14, 152-163. https://doi.org/10.1038/embor.2012.219

[5] Gold, M.S. and Gebhart, G.F. (2010) Nociceptor Sensitization in Pain Pathogenesis. Nature Medicine, 16, 1248-1257. https://doi.org/10.1038/nm.2235

[6] Alessandri-Haber, N., Dina, O.A., Chen, X. and Levine, J.D. (2009) TRPC1 and TRPC6 Channels Cooperate with TRPV4 to Mediate Mechanical Hyperalgesia and Nociceptor Sensitization. Journal of Neuroscience, 29, 6217-6228. https://doi.org/10.1523/JNEUROSCI.0893-09.2009

[7] Liedtke, W. and Friedman, J.M. (2003) Abnormal Osmotic Regulation in Trpv4 ${ }^{-1}$ Mice. Proceedings of the National Academy of Sciences of the United States of America, 100, 13698-13703. https://doi.org/10.1073/pnas.1735416100

[8] Liedtke, W., Tobin, D.M., Bargmann, C.I. and Friedman, J.M. (2003) Mammalian TRPV4 (VR-OAC) Directs Behavioral Responses to Osmotic and Mechanical Stimuli in Caenorhabditis elegans. Proceedings of the National Academy of Sciences of the United States of America, 2, 14531-14536.

https://doi.org/10.1073/pnas.2235619100

[9] Liedtke, W. (2005) TRPV4 Plays an Evolutionary Conserved Role in the Transduction of Osmotic and Mechanical Stimuli in Live Animals. The Journal of Physiology, 567, 53-58. https://doi.org/10.1113/jphysiol.2005.088963

[10] Vriens, J., Watanabe, H., Janssens, A., Droogmans, G., Voets, T. and Nilius, B. (2004) Cell Swelling, Heat, and Chemical Agonists Use Distinct Pathways for the Activation of the Cation Channel TRPV4. Proceedings of the National Academy of 
Sciences of the United States of America, 101, 396-401. https://doi.org/10.1073/pnas.0303329101

[11] Everaerts, W., Nilius, B. and Owsianik, G. (2010) The Vanilloid Transient Receptor Potential Channel TRPV4: From Structure to Disease. Progress in Biophysics and Molecular Biology, 103, 2-17. https://doi.org/10.1016/j.pbiomolbio.2009.10.002

[12] Delany, N.S., Hurle, M., Facer, P., Alnadaf, T., Plumpton, C., Kinghorn, I., See, C.G., Costigan, M., Anand, P., Woolf, C.J., Crowther, D., Sanseau, P. and Tate, S.N. (2001) Identification and Characterization of a Novel Human Vanilloid Receptor-Like Protein, VRL-2. Physiological Genomics, 4, 165-174. https://doi.org/10.1152/physiolgenomics.2001.4.3.165

[13] Watanabe, H., Vriens, J., Prenen, J., Droogmans, G., Voets, T. and Nilius, B. (2003) Anandamide and Arachidonic Acid Use Epoxyeicosatrienoic Acids to Activate TRPV4 Channels. Nature, 424, 434-438. https://doi.org/10.1038/nature01807

[14] Suzuki, M., Hirao, A. and Mizuno, A. (2003) Microtubule-Associated [Corrected] Protein 7 Increases the Membrane Expression of Transient Receptor Potential Vanilloid 4 (TRPV4). The Journal of Biological Chemistry, 278, 51448-51453. https://doi.org/10.1074/jbc.M308212200

[15] Strotmann, R., Schultz, G. and Plant, T.D. (2003) $\mathrm{Ca}^{2+}$-Dependent Potentiation of the Nonselective Cation Channel TRPV4 Is Mediated by a C-Terminal Calmodulin Binding Site. The Journal of Biological Chemistry, 278, 26541-26549. https://doi.org/10.1074/jbc.M302590200

[16] Gagnon, K.B., England, R. and Delpire, E. (2006) Volume Sensitivity of Cation-Cl ${ }^{-}$ Cotransporters Is Modulated by the Interaction of Two Kinases: Ste20-Related Proline-Alanine-Rich Kinase and WNK4. American Journal of Physiology-Cell Physiology, 290, C134-C142. https://doi.org/10.1152/ajpcell.00037.2005

[17] Shin, S.H., Lee, E.J., Chun, J., Hyun, S. and Kang, S.S. (2015) Phosphorylation on TRPV4 Serine 824 Regulates Interaction with STIM1. The Open Biochemistry Journal, 9, 24-33. https://doi.org/10.2174/1874091X01509010024

[18] Cuajungco, M.P., Grimm, C., Oshima, K., D’Hoedt, D., Nilius, B., Mensenkamp, A.R., Bindels, R.J., Plomann, M. and Heller, S. (2006) PACSINs Bind to the TRPV4 Cation Channel. PACSIN 3 Modulates the Subcellular Localization of TRPV4. The Journal of Biological Chemistry, 281, 18753-18762. https://doi.org/10.1074/jbc.M602452200

[19] Palacio-Mancheno, P.E., Evashwick-Rogler, T.W., Laudier, D.M., Purmessur, D. and Iatridis, J.C. (2018) Hyperosmolarity Induces Notochordal Cell Differentiation with Aquaporin3 Upregulation and Reduced N-Cadherin Expression. Journal of Orthopaedic Research, 36, 788-798. https://doi.org/10.1002/jor.23715

[20] Sokabe, T., Fukumi-Tominaga, T., Yonemura, S., Mizuno, A. and Tominaga, M. (2010) The TRPV4 Channel Contributes to Intercellular Junction Formation in Keratinocytes. The Journal of Biological Chemistry, 285, 18749-18758. https://doi.org/10.1074/jbc.M110.103606

[21] Becker, D., Bereiter-Hahn, J. and Jendrach, M. (2009) Functional Interaction of the Cation Channel Transient Receptor Potential Vanilloid 4 (TRPV4) and Actin in Volume Regulation. European Journal of Cell Biology, 88, 141-152. https://doi.org/10.1016/j.ejcb.2008.10.002

[22] Fernandes, J., Lorenzo, I.M., Andrade, Y.N., Garcia-Elias, A., Serra, S.A., Fernandez-Fernandez, J.M. and Valverde, M.A. (2008) IP3 Sensitizes TRPV4 Channel to the Mechano- and Osmotransducing Messenger 5'-6'-Epoxyeicosatrienoic Acid. Journal of Cell Biology, 181, 143-155. https://doi.org/10.1083/jcb.200712058 
[23] Fusi, C., Materazzi, S., Minocci, D., Maio, V., Oranges, T., Massi, D. and Nassini, R. (2014) Transient Receptor Potential Vanilloid 4 (TRPV4) Is Downregulated in Keratinocytes in Human Non-Melanoma Skin Cancer. Journal of Investigative Dermatology, 134, 2408-2417. https://doi.org/10.1038/jid.2014.145

[24] Doudna, J.A. and Charpentier, E. (2014) The New Frontier of Genome Engineering with CRISPR-Cas9. Science, 346, 1258096.

https://doi.org/10.1126/science.1258096

[25] Fiorio Pla, A., Ong, H.L., Cheng, K.T., Brossa, A., Bussolati, B., Lockwich, T., Paria, B., Munaron, L. and Ambudkar, I.S. (2012) TRPV4 Mediates Tumor-Derived Endothelial Cell Migration via Arachidonic Acid-Activated Actin Remodeling. Oncogene, 31, 200-212. https://doi.org/10.1038/onc.2011.231

[26] Vriens, J., Janssens, A., Prenen, J., Nilius, B. and Wondergem, R. (2004) TRPV Channels and Modulation by Hepatocyte Growth Factor/Scatter Factor in Human Hepatoblastoma (HepG2) Cells. Cell Calcium, 36, 19-28.

https://doi.org/10.1016/j.ceca.2003.11.006

[27] Ran, F.A., Hsu, P.D., Wright, J., Agarwala, V., Scott, D.A. and Zhang, F. (2013) Genome Engineering Using the CRISPR-Cas9 System. Nature Protocols, 8, 2281-2308. https://doi.org/10.1038/nprot.2013.143

[28] Shin, S.H., Lee, E.J., Hyun, S., Chun, J., Kim, Y. and Kang, S.S. (2012) Phosphorylation on the Ser 824 Residue of TRPV4 Prefers to Bind with F-Actin than with Microtubules to Expand the Cell Surface Area. Cellular Signalling, 24, 641-651. https://doi.org/10.1016/j.cellsig.2011.11.002

[29] Lee, W.H., Choong, L.Y., Mon, N.N., Lu, S., Lin, Q., Pang, B., Yan, B., Krishna, V.S., Singh, H., Tan, T.Z., Thiery, J.P., Lim, C.T., Tan, P.B., Johansson, M., Harteneck, C. and Lim, Y.P. (2016) TRPV4 Regulates Breast Cancer Cell Extravasation, Stiffness and Actin Cortex. Scientific Reports, 6, 27903. https://doi.org/10.1038/srep27903

[30] Adapal, R.K., Thoppil, R.J., Ghosh, K., Cappelli, H.C., Dudley, A.C., Paruchuri, S., Keshamouni, V., Klagsbrun, M., Meszaros, J.G., Chilian, W.M., Ingber, D.E. and Thodeti, C.K. (2016) Activation of Mechanosensitive Ion Channel TRPV4 Normalizes Tumor Vasculature and Improves Cancer Therapy. Oncogene, 35, 314-322. https://doi.org/10.1038/onc.2015.83

[31] Monteith, G.R., Davis, F.M. and Roberts-Thomson, S.J. (2012) Calcium Channels and Pumps in Cancer: Changes and Consequences. The Journal of Biological Chemistry, 287, 31666-31673. https://doi.org/10.1074/jbc.R112.343061

[32] Sharma, S., Goswami, R., Zhang, D.X. and Rahaman, S.O. (2018) TRPV4 Regulates Matrix Stiffness and TGF $\beta 1$-Induced Epithelial-Mesenchymal Transition. Journal of Cellular and Molecular Medicine, 23, 761-774. https://doi.org/10.1111/jcmm.13972 